Z. klin. Chem. u. klin. Biochem.

7. Jg., S. 592-598, November 1969

\title{
Zuverlässigkeit der Bestimmung der 17-Ketosteroide mittels Gaschromatographie und Korrelation mit den Werten erhalten nach der Methode von ZiMmERMANN
}

\author{
Von H. Gleispach, Barbara Podolski¹), W. Hohenwallner und H. Berger \\ Aus der Universitäts-Kinderklinik Innsbruck (Vorstand: Prof. Dr. H. Berger)
}

(Eingegangen am 27. Juni 1969)

\begin{abstract}
Die vorliegende Arbeit befaßt sich mit dem Vergleich der Werte, die für die 17-Ketosteroide einerseits bei Verwendung der ZimmermannReaktion zur Summenbestimmung und andererseits bei Verwendung der Gaschromatographie zur Bestimmung der Einzelmetabolite erhalten werden. Es wird die Genauigkeit der gaschromatographischen Steroidbestimmung im Routinebetrieb diskutiert. Die Einzelschritte der Bestimmung werden genau untersucht. Hierbei kann festgestellt werden, daß die Unterschiede in den erhaltenen Summen auf Substanzen zurückzuführen sind, die im wesentlichen erst bei der heißen Säurehydrolyse entstehen, mit Äther extrahierbar sind und mit Dinitrobenzol reagieren, aber keine Steroide darstellen. Auch konnten wir zeigen, daß die Genauigkeit der gaschromatographischen Bestimmung nach Erstellung von Eichkurven bei $\pm 10 \%$ liegt.
\end{abstract}

The reliability of the determination of 17-ketosteroids by gas-chromatography and the correlation of the values with those obtained by the method of ZIMMERMANN

In the present work, the values obtained for the determination of total 17-ketosteroids by the Zimmermann-Reaction were compared with those for the individual metabolites determined by gas-chromatography. The accuracy of the routine measurement of steroids by gaschromatography is discussed; the separate steps of the determination were studied in detail. It was thus shown that the differences in the final totals were caused by substances that are produced by hot acid hydrolysis. These substances can be extracted with ether, they react with dinitrobenzene, but they are not steroids. It was also shown by the preparation of calibration curves, that the accuracy of the gaschromatographic determination is $\pm 10 \%$.

Als klinische Routinemethode zur Bestimmung der 17-Ketosteroide hat die Methode von ZimmermanN auch heute noch die größte Bedeutung, dennoch rücken die Methoden zur Bestimmung von Einzelmetaboliten immer mehr in das Blickfeld je mehr sie vereinfacht werden.

Von den chromatographischen Trennmethoden werden vor allem drei zur Bestimmung der Steroidhormone angewandt.

1. Papierchromatographie

2. Dünnschichtchromatographie

3. Gaschromatographie

Die Säulenchromatographie (1) wird nur bei einigen speziellen Trennproblemen verwendet, da sie recht genaue Ergebnisse liefert, für eine klinische Routineuntersuchung aber zu zeitraubend ist. Größere Bedeutung dürfte die von CREMER und Mitarbeitern (2) entwickelte Dünnfilmchromatographie exlangen, bei der in Kombination mit einem Zeiss-Densitometer Mengen von $10 \mathrm{pg}$ noch nachgewiesen werdden können. Die Entwicklung eines solchen Chromatogramms dauert weit weniger lang als die eines herkömmlichen Dünnschichtchromatogramms, so daß diese Methode vielleicht einen idealen Screening-Test darstellen oder in Kombination mit einer der anderen Trennmethoden eine rasche Bestimmung ermöglichen wird.

1) Die Untersuchungen wurden von Barbara PoDolski im Rahmen ihrer Doktorarbeit durchgeführt.
Von den oben angeführten Bestimmungsmethoden für die Harnsteroide stellt die Gaschromatographie in Kombination mit einer raschen. Vortrennung und Reinigung mittels Säulenchromatographie bzw. Dünnschichtchromatographie wohl die exakteste und auch rascheste Methode dar.

Vergleicht man die Werte, die bei der Bestimmung der 17-Ketosteroide mit der Methode nach ZimmermanN (3) erhalten vurden, mit der Summe der gaschromatographisch ermittelten Einzelmetabolite der 17-Ketosteroide, so findet man beträchtliche Unterschiede. Vor allem aber stehen die beiden Werte in keinem konstanten Verhältnis zueinander. Außerdem fällt auf, daß einerseits im Kindesalter die Differenzen größer sind als im Erwachsenenalter (Tab. 1a), andererseits bei Belastungstests nach Lloyd und Mitarbeitern (4) (30 IE ACTH $/ \mathrm{m}^{2}$ Körperfläche in einer Infusion während 6 Stunden, Harnsammeln ab Beginn der Infusion, dann durch 14 Tage täglich 0,033 mg Dexamethason $/ \mathrm{kg}$, nach dem 7. Tag Harnsammeln, dann während der Dexamethasongaben $4 \times 1500 \mathrm{IE}$ Choriongonadotropine $/ \mathrm{m}^{2}$ Körperoberfläche jeden 2. Tag und wieder Harnsammeln) mittels der Gaschromatographie ein stärkeres Ansprechen auf die Belastung festzustellen ist als nach der Methode von ZimmermanN (Tab. 1b).

Wir befaßten uns mit der Frage, worauf diese Unterschiede in den nach beiden Methoden erhaltenen Werten zurückzuführen sind und wie genau die von uns durch- 


\section{Blutzuckerbestimmung enzymatisch}
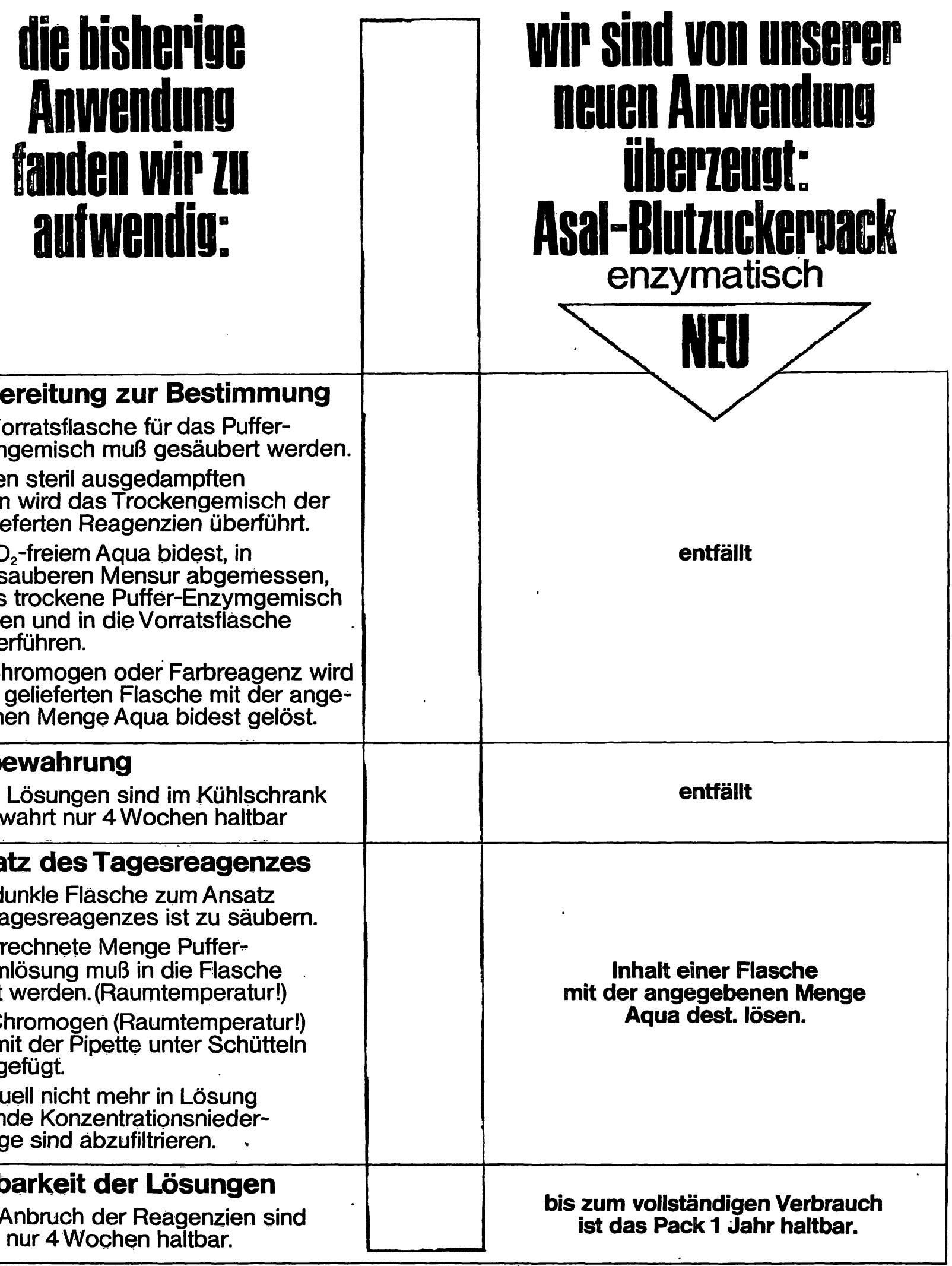

1. Vorbereitung zur Bestimmung

a. eine Vorratsflasche für das Puffer-

Enzymgemisch muß gesäubert werden.

in einen steril ausgedampften

Kolben wird das Trockengemisch der angelieferten Reagenzien überführt.

mit $\mathrm{CO}_{2}$-freiem Aqua bidest, in

einer sauberen Mensur abgemessen,

ist das trockene Puffer-Enzymgemisch

zu lösen und in die Vorratsflasche

zu überführen.

b. das Chromogen oder Farbreagenz wird in der gelieferten Flasche mit der angegebenen Menge Aqua bidest gelöst.

\section{Aufbewahrung}

Beide Lösungen sind im Kühlschrank aufbewahrt nur 4 Wochen haltbar

\section{Ansatz des Tagesreagenzes}

a. eine dunkle Flasche zum Ansatz des Tagesreagenzes ist zu säubern.

b. die errechnete Menge PufferEnzymlösung muß in die Flasche gefüllt werden. (Raumtemperatur!)

c. das Chromogen (Raumtemperatur!) wird mit der Pipette unter Schütteln hinzugefügt.

d. eventuell nicht mehr in Lösung gehende Konzentrationsniederschläge sind abzufiltrieren.

\section{Haltbarkeit der Lösungen} nach Anbruch der Reagenzien sind diese nur 4 Wochen haltbar.

Chemische Fabrik Asal 6231 Schwalbach/Taunus Lauenburger Straße 5 Telefon (06196) 3031

Überzeugen Sie sich.

Fordem Sie bitte Muster und Informationsmaterial an! 

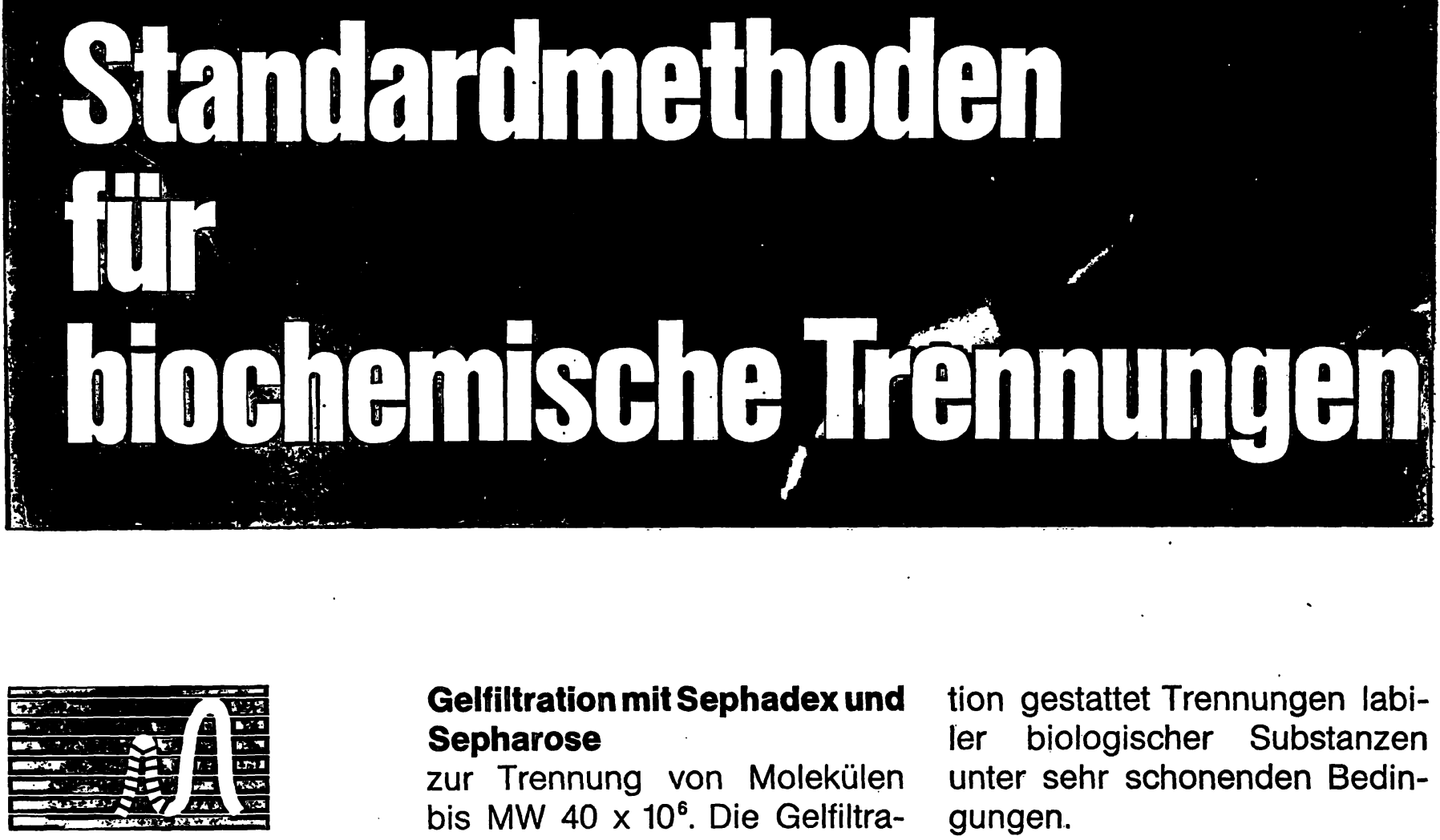

Gelfiltration mit Sephadex und Sepharose

zur Trennung von Molekülen bis MW $40 \times 10^{6}$. Die Gelfiltra-

tion gestattet Trennungen labiler biologischer Substanzen unter sehr schonenden Bedingungen.

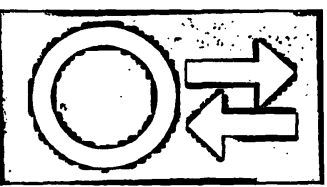

Ionenaustauscher-Chromato-

einigen. Charakteristisch sind graphie mit Sephadex-Ionenaustauschern, die die Vorzüge von CelluloseundKunstharzaustauschern verhohe Kapazität, niedrige unspezifische Adsorption und ausgezeichnete Reproduzierbarkeit.

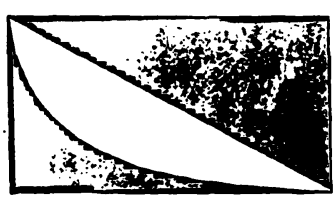

Verteilung in wäßrigen Pha-

zur Fraktionierung sehr hochsen-Systemen mit Dextran und Dextran-Derivaten molekularer Stoffe wie Viren, Nukleinsäuren und Zellpartikeln unter sehr milden Bedingungen.

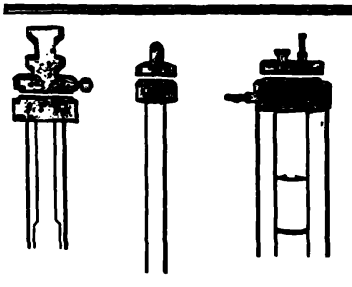

\section{Chromatographierohre}

Unsere Chromatographierohre, die wir speziell für Gelfiltration und lonenaustausch-Chromato-

graphie entwickelt haben, ermöglichen reproduzierbare Trennresultate. Sie stehen Ihnen in großer Auswahl mit diversem Zubehör zur Verfügung.

\section{Literaturdienst}

Als Hilfe für Wissenschaftler geben wir einen umfassenden Literaturdienst heraus. Eine jährlich erscheinende Referenzliste enthält etwa 1000 neue
Literaturstellen. Bitte schreiben Sie uns, wenn wir Sie in unseren Verteiler aufnehmen sollen. Fordern Sie auch Broschüren über unsere Separationsprodukte und das Literaturverzeichnis an. 
geführte gaschromatographische Bestimmungsmethode ist. Die Genauigkeit der Zimmermann-Reaktion soll in dieser Arbeit nur insoweit zur Diskussion gestellt werden, als sie die in unserem Laboratorium erhaltenen Werte betrifft, da ja über die verschiedenen Genauigkeitskriterien der Zimmermann-Methode schon ausführlich von berufener Stelle (3) berichtet wurde.

\section{Methodik}

\section{Untersuchungsgang}

\section{Methode nach ZIMMERMANN}

1. Streubreite bei Mehrfachbestimmungen durch Veränderung von Temperatur und Reaktionsdauer sowohl bei der Hydrolyse als auch bei der Farbreaktion.

2. Erstellung von Eichkurven für die einzelnen gaschromatographisch bestimmten Steroide auch nach der Methode von ZimMERMANN (Abb. 1).

Tab. $1 \mathrm{a}$

Vergleich der nach ZIMMERMANN bestimmten 17-Ketosteroide mit der Summe der gaschromatographisch erhaltenen Werte

\begin{tabular}{|c|c|c|c|c|c|}
\hline Name & Alter & $\begin{array}{c}\text { Ge- } \\
\text { schlech }\end{array}$ & $\begin{array}{c}\text { Gesamt-17- } \\
\text { Ketosteroide } \\
\text { nach } \\
\text { ZimMERMANN } \\
\text { (mg/Tag) }\end{array}$ & $\begin{array}{c}\text { gaschro } \\
\text { (mg/Tag) }\end{array}$ & $\begin{array}{c}\text { 1atographisch } \\
\text { (in Pro- } \\
\text { zenten nach } \\
\text { ZIMMER- } \\
\text { MANN) }\end{array}$ \\
\hline $\begin{array}{l}\text { M. D. } \\
\text { N. I. } \\
\text { L. E. } \\
\text { R. A. } \\
\text { W. E. } \\
\text { H. O. } \\
\text { T. A. } \\
\text { W. A. } \\
\text { S. T. } \\
\text { K. U. } \\
\text { S. M. } \\
\text { C. A. } \\
\text { P. E. } \\
\text { L. A. } \\
\text { R. A. } \\
\text { L. A. } \\
\text { G. H. }\end{array}$ & $\begin{array}{c}12 \text { Tage } \\
7 \text { Jahre } \\
10 \text { Jahre } \\
10 \text { Jahre } \\
10 \text { Jahre } \\
10 \text { Jahre } \\
11 \text { Jahre } \\
11 \text { Jahre } \\
12 \text { Jahre } \\
13 \text { Jahre } \\
14 \text { Jahre } \\
15 \text { Jahre } \\
15 \text { Jahre } \\
25 \text { Jahre } \\
28 \text { Jahre } \\
29 \text { Jahre } \\
31 \text { Jahre }\end{array}$ & 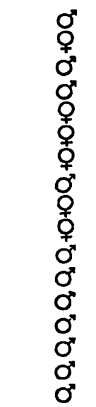 & $\begin{array}{r}1,00 \\
1,73 \\
4,66 \\
3,97 \\
2,44 \\
1,11 \\
2,16 \\
3,89 \\
3,45 \\
3,25 \\
5,10 \\
7,38 \\
3,83 \\
6,70 \\
18,20 \\
20,60 \\
17,30\end{array}$ & $\begin{array}{r}0,14 \\
0,26 \\
0,81 \\
1,78 \\
0,65 \\
0,34 \\
0,55 \\
0,79 \\
1,23 \\
2,43 \\
1,69 \\
4,23 \\
1,70 \\
5,48 \\
16,80 \\
5,75 \\
15,70\end{array}$ & $\begin{array}{l}14 \\
15 \\
18 \\
45 \\
26 \\
31 \\
39 \\
20 \\
36 \\
75 \\
30 \\
57 \\
45 \\
82 \\
92 \\
36 \\
91\end{array}$ \\
\hline
\end{tabular}

II. Gaschromatographic

1. Eichkurven für sämtliche zu bestimmenden 'Steroide (in steigender Konzentration dem Harn zugegeben).

2. Streubreite bei Mehrfachbestimmungen.

3. Einfluß verschiedener Silylierverfahren.

a) Bistrimethylsilylacetamid (BSA) - Hexamethyldisilazan (HMDS) + Trimethylchlorsilan (TMCS).

b) Einfluß der HMDS- und TMCS-Konzentration (Tab. 3a).

c) Einfluß der Reaktionsdauer (Tab. 3 b).

d) Einfluß der Reaktionstemperatur (Tab. 3c).

III. Probenaufbereitung

1. Verschiedene Hydrolysenmethoden (Tab. 4 und 5).

a) Enzymatische Spaltung.

b) $\beta$-Glucuronidase-Spaltung und anschließende Solvolyse bei $\mathrm{pH}=1$ und kontinuierliche Atherextraktion.

c) Hydrolyse durch $\mathrm{HCl}$ bzw. $\mathrm{H}_{2} \mathrm{SO}_{4}$.

2. Verfolgen jedes Einzelschritts der Hydrolyse, der Extraktion und Reinigung bei einigen Harnen nach beiden Methoden (Tab. 5).

\section{Arbeitsmethode}

I. Methode nach ZimmermanN

\section{Hydrolyse}

Bei einer Bestimmung der Gesamt-17-Ketosteroide verwendet man im allgemeinen die heiße Säurehydrolyse zur Spaltung der im Harn enthaltenen Steroidkonjugate. Bei der ZimmermannMethode (3) versetzt man $25 \mathrm{ml}$ des 24-Stdn.-Harnes mit $2,5 \mathrm{ml}$ konz. $\mathrm{HCl}$ und kocht $20 \mathrm{Min}$. unter Rückfluß. Nach dem.Abkühlen wird $3 \mathrm{mal}$ mit Äther extrahiert. Nach der Vorschrift von ZIMMERMANN und Pontius (5) wetden $10 \mathrm{ml}$ Harn mit $1 \mathrm{ml}$ konz. $\mathrm{HCl}$ und $1 \mathrm{ml} 10$ proz. Kupfersulfatlösung $20 \mathrm{Min}$. unter Rückfluß gekocht. BIRKET-SMITH (6) kochen $10 \mathrm{ml}$ Harn mit $1 \mathrm{ml} 40$ proz. $\mathrm{H}_{2} \mathrm{SO}_{4} 25 \mathrm{Min}$. unter Rückfluß. Alle drei Verfahren wurden bei uns durchgeführt (Tab. 5).

\section{Extraktion und Reinigung}

Anschließend an die Hydrolyse wird im Scheidetrichter $3 \mathrm{mal}$ mit je $50 \mathrm{ml}$ Ather extrahiert. Die Extrakte werden mit $50 \mathrm{ml}$

Tab. $1 \mathrm{~b}$

17-Ketosteroidausscheidung ( $\mu \mathrm{g} / \mathrm{Tag}$ ) vor und nach Gabe verschiedener Hormonpräparate

\begin{tabular}{|c|c|c|c|c|c|c|c|c|c|c|c|c|c|}
\hline \multirow[b]{2}{*}{ Proband } & \multirow[b]{2}{*}{ Belastung } & \multirow[b]{2}{*}{ 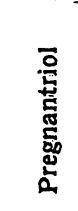 } & \multirow[b]{2}{*}{ 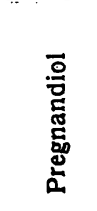 } & \multirow[b]{2}{*}{ 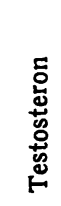 } & \multirow[b]{2}{*}{ 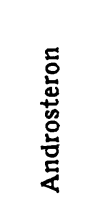 } & \multirow{2}{*}{ 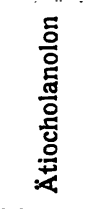 } & \multirow[b]{2}{*}{ 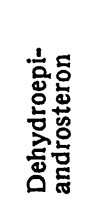 } & \multirow[b]{2}{*}{ 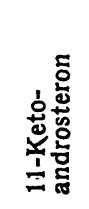 } & \multirow{2}{*}{ 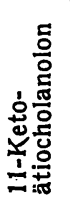 } & \multirow{2}{*}{ 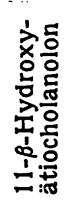 } & \multirow{2}{*}{ 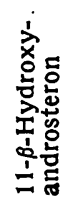 } & \multicolumn{2}{|c|}{$\begin{array}{l}\text { Gesamt-17- } \\
\text { Ketosteroide }\end{array}$} \\
\hline & & & & & & & & & & & & 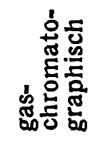 & 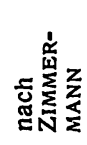 \\
\hline L. $\stackrel{\sigma^{\infty}}{\mathrm{K}}$. & $\begin{array}{l}\text { vor } \\
\text { ACTH } 60 \text { IE } \\
\text { Dexamethason }\end{array}$ & $\begin{array}{r}520 \\
1720\end{array}$ & $\begin{array}{l}1840 \\
2300\end{array}$ & $\begin{array}{r}35 \\
115\end{array}$ & $\begin{array}{r}605 \\
3780\end{array}$ & $\begin{array}{l}3200 \\
\mathbf{7 7 5 0}\end{array}$ & $\begin{array}{r}480 \\
3700\end{array}$ & $\begin{array}{r}520 \\
1700\end{array}$ & $\begin{array}{l}394 \\
815\end{array}$ & $\begin{array}{l}364 \\
394\end{array}$ & $\begin{array}{l}550 \\
320\end{array}$ & $\begin{array}{r}5750 \\
18460\end{array}$ & $\begin{array}{l}20600 \\
23300\end{array}$ \\
\hline 27 Jahre & $\begin{array}{l}2,5 \mathrm{mg} / \mathrm{Tag} \\
\text { HCG } 4 \times 3000 \mathrm{IE} \\
\text { u. Dexamethason } \\
2,5 \mathrm{mg} \text { tägl. }\end{array}$ & $\begin{array}{r}340 \\
1130\end{array}$ & $\begin{array}{l}290 \\
666\end{array}$ & $\begin{array}{l}27 \\
35\end{array}$ & $\begin{array}{l}535 \\
727\end{array}$ & $\begin{array}{l}1040 \\
1305\end{array}$ & $\overline{192}$ & $\begin{array}{r}90 \\
.287\end{array}$ & 24 & 二 & 二 & $\begin{array}{l}1690 \\
2500\end{array}$ & $\begin{array}{r}8900 \\
10800\end{array}$ \\
\hline N. ${ }^{+} \mathrm{R}$. & $\begin{array}{l}\text { vor } \\
\text { ACTH } 20 \text { IE }\end{array}$ & $\begin{array}{r}15 \\
632\end{array}$ & $\begin{array}{r}28 \\
292\end{array}$ & $\overline{18}$ & $\begin{array}{r}78 \\
332\end{array}$ & $\begin{array}{r}26 \\
128\end{array}$ & $\begin{array}{r}5 \\
64\end{array}$ & $\begin{array}{r}14 \\
346\end{array}$ & $\begin{array}{l}100 \\
768\end{array}$ & $\begin{array}{r}22 \\
512\end{array}$ & $\begin{array}{r}17 \\
296\end{array}$ & $\begin{array}{r}262 \\
2440\end{array}$ & $\begin{array}{l}1720 \\
5770\end{array}$ \\
\hline \multirow[t]{2}{*}{13 Jahre } & \multirow{2}{*}{$\begin{array}{l}\text { Dexamethason } \\
0,5 \mathrm{mg} / \mathrm{Tag} \\
\text { HCG } 4 \times 1000 \mathrm{IE}\end{array}$} & 5 & 22 & 5 & 42 & 10 & 10 & 6 & 12 & 5 & 5 & 85 & 1000 \\
\hline & & 120 & 60 & 5 & 78 & 99 & 10 & 79 & 322 & 38 & 125 & 751 & 2600 \\
\hline H. $\mathrm{P}$. & $\begin{array}{l}\text { vor } \\
\text { ACTH } 50 \text { IE }\end{array}$ & $\begin{array}{r}470 \\
2200\end{array}$ & $\begin{array}{r}.522 \\
2630\end{array}$ & $\begin{array}{l}20 \\
30\end{array}$ & $\begin{array}{r}535 \\
3190\end{array}$ & $\begin{array}{l}1920 \\
3960\end{array}$ & $\begin{array}{r}221 \\
1370\end{array}$ & $\begin{array}{r}257 \\
1670\end{array}$ & $\begin{array}{l}386 \\
590\end{array}$ & $\begin{array}{l}106 \\
480\end{array}$ & $\begin{array}{l}180 \\
665\end{array}$ & $\begin{array}{r}3605 \\
11920\end{array}$ & $\begin{array}{l}10800 \\
14370\end{array}$ \\
\hline \multirow[t]{2}{*}{29 Jahre } & $\begin{array}{c}\text { Dexamethason } \\
2,0 \mathrm{mg} / \text { tägl. }\end{array}$ & 595 & 550 & 一 & 195 & 336 & - & 54 & - & - & - & 584 & 4370 \\
\hline & $\begin{array}{l}\text { HCG } 4 \times 2500 \text { IE } \\
\text { u. Dexamethason } \\
2,0 \mathrm{mg} / \text { tägl. }\end{array}$ & 2100 & 10000 & 23 & 690 & 2380 & 129 & - & - & - & - & 3199 & 6480 \\
\hline
\end{tabular}


10proz. $\mathrm{NaOH}$ und $2 \mathrm{mal} \mathrm{mit} \mathrm{je} 50 \mathrm{~m} l$ Wasser gewaschen, vereinigt und der Gesamtextrakt wird zur Trockene eingedampft. Zuvor wird der Ätherextrakt mit Natriumsulfat getrocknet und filtriert oder nach Abdampfen des Äthers nochmals mit etwas Methanol versetzt und das restliche Wasser mit dem Methanol entfernt. Nach unseren Erfahrungen sind die Verluste nach dem letzten Verfahren geringer.

\section{Farbreaktion}

Nach Zimmermann handelt es sich um eine Addition des Carbeniat-Ions des Ketons in Gegenwart von Alkali an Dinitrobenzol. Durch überschüssiges $m$-Dinitrobenzol wird die für Formel $I$ angenommene Bindung zu einer Doppelbindung dehydriert und es entsteht ein resonanzfähiges Elektronensystem mit charakteristischer Absorptionsbande.<smiles>CC1CC(C)C(C)C1=O</smiles><smiles>[AlH2]</smiles><smiles>CC1CC(c2ccc([N+](=O)[O-])cc2[N+](=O)[O-])C(Cl)C1C</smiles><smiles>[CH][CH]</smiles><smiles>CC1CC(c2ccc([N+](=O)[O-])cc2[N+](=O)[O-])C(=O)C1C</smiles>

(I) $\mid \begin{aligned} & m \text {-Dinitro- } \\ & \text { benzol }\end{aligned}$<smiles>CC1CC(=C2C=CC(=[N+]([O-])[O-])C=C2[N+](=O)[O-])C(=O)C1C</smiles>

Der entstandene Farbkomplex wird dann mit Äther extrahiert und bei $510 \mathrm{~nm}$ gemessen. Vielfach wird auch empfohlen, bei zwei Wellenlängen zu messen und den Wert nach der Formel von Gibson und Evelys (8) zu korrigieren oder bei drei Wellenlängen zu messen und Störfaktoren nach der von ALLEN (9) bzw. der von Brown (10) eingeführten Formel zu eliminieren. Wir arbeiten bei unseren Untersuchungen nach der Vorschrift von Zimmermann (3). Der Rückstand des Harnextraktes wird in $5 \mathrm{ml}$ Äthanol gelöst, dann werden folgende Lösungen hergestellt.

1. Harnextraktlösung: $1 \mathrm{~m} l$ Harnextrakt $+1 \mathrm{~m} l$ Äthanol $+2 \mathrm{~m} l$ 3proz. Dinitrobenzol $+2 \mathrm{~m} / 3 \mathrm{~N} \mathrm{KOH}$.

2. Harnextraktblindlösung: $1 \mathrm{~m} l$ Harnextrakt $+3 \mathrm{~m} l$ Åtha$\mathrm{nol}+2 \mathrm{~m} / 3 \mathrm{~N} \mathrm{KOH}$.

3. Reagenzienblindlösung: $2 \mathrm{~m} l$ Äthanol $+2 \mathrm{ml} 3 \mathrm{~N} \mathrm{KOH}+2 \mathrm{ml}$ 3proz. Dinitrobenzol in Äthanol.

Nach dem Einpipettieren wird geschüttelt, dann 90 Min. im Dunkeln bei $25^{\circ}$ stehengelassen. Nach Beendigung der Reaktion wird der Farbkomplex mit $5 \mathrm{ml}$ Äther extrahiert und gegen Äther als Vergleichislösung bei $510 \mathrm{~nm}$ gemessen.

\section{Gaschromatographie}

Bei der gaschromátographischen Bestimmung stellt die Präparation die Hauptarbeit dar. Voraussetzung für eine einwandfreie gaschromatographische Bestimmung ist ein möglichst reiner Harnextrakt, wobei noch besonderes Augenmerk darauf zu richten ist, die Bildung von Artefakten bei der Präparation möglichst zu vermeiden.

\section{Hydrolyse und Extraktion}

Mit dem Einfluß, den die verschiedenen Hydrolyseverfahren auf die Analysengenauigkeit ausüben, setzten sich vor allem Curtius und Mitarbeiter (11) auseinander. Sie stellten fest, daß man die besten Ergebnisse erhält, wenn man zuerst die an Glucuronsäure gebundenen Steroide enzymatisch mit $\beta$-Glucuronidase spaltet, die freien Steroide mit Äther extrahiert und den Harn dann einer Solvolyse bei Raumtemperatur und kontiniuerlicher Äther- extraktion unterzieht. Zur Spaltung der Glucuronide wird ein aliquoter Teil des Harnes mit Essigsäure bzw. Natronlauge auf $\mathrm{pH}=6$ gebracht. Dann wird, auf je $100 \mathrm{ml}$ Harn berechnet, $30 \mathrm{ml}$ 1N Natriumacetatpuffer $\mathrm{pH}=6$ zugegeben und dann 10000 Fishman-Einheiten $\beta$-Glucurónidase und einige Tropfen Chloroform. Das Chloroform dient als Bakteriostaticum und zur Aktivierung der $\beta$-Glucuronidase. Nun wird der Harn während $24 \mathrm{Stdn}$. bei $38^{\circ}$ inkubiert, abgekühlt und dann $3 \mathrm{mal}$ mit Äther extrahiert. Für die Extraktion haben wir uns ein von Curtrus mitgeteiltes Verfahren zu eigen gemacht: 3 Schütteltrichter werden hintereinander aufgestellt und der Harn jeweils nach dex Extraktion von einem in den anderen Schütteltrichter überführt. In gleicher Weise werden auch die Waschflüssigkeiten hindurchgeführt; auf diese Weise werden die bei der Extraktion und beim Waschen verursachten Verluste auf ein Minimum reduziert. Um den Ätherextrakt von sauren Substanzen zu befreien wird der Äther $2 \mathrm{mal}$ mit $2 \mathrm{~N}$ Natriumcarbonat gewaschen, dann wird $3 \mathrm{mal}$ mit Wasser neutral gewaschen und der Äther zur Trockene eingedampft. Um dem Äther anhaftendes Wasser zu entfernen, wird etwas Methanol zugefügt und nochmals eingeengt.

Die bei der Ätherextraktion anfallende wäßrige Phase wird mit 6N Schwefelsäure auf $\mathrm{pH}=1$ angesäuert und in einem Extraktor für leichte Lösungsmittel während zweiet Tage kontinuierlich mit Äther extrahiert. Anschließnend wird der Ätherextrakt wieder

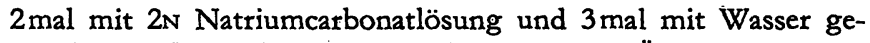
waschen und mit dem Rückstand -vom ersten Ätherextrakt vereinigt und abermals zur Trockene eingedampft.

\section{Säulenchromatographie}

Zur Reinigung und Vortrennung führen wir eine Säulenchromatographie an Aluminiumoxid (Woelm neutral, Aktivitätsstufe III) durch. In eine Säule mit $1,8 \mathrm{~mm}$ Innendurchmesser gibt man $5 \mathrm{ml}$ absolutes Benzol und füllt $4 \mathrm{~g}$ Aluminiumoxid Luftblasen-frei ein. Das überstehende Benzol wird bis knapp über die Aluminiumoxidoberfläche abgelassen. Nun wird der Steroidextrakt mit $3 \mathrm{mal} 3 \mathrm{ml}$ Benzol aufgebracht. Mit weiteren $11 \mathrm{~m} l$ Benzol wird nachgewaschen. Diese ersten $20 \mathrm{~m} l$ Benzol werden verworfen. Dann wird die erste Fraktion aufgefangen. Hierfür wird zuerst mit $40 \mathrm{~m} l$ Benzol und anschließend mit $30 \mathrm{~m} l$ Benzol $+0,2 \mathrm{ml}$ Äthanol eluiert. In dieser Fraktion befinden sich die drei 11-Desoxy-17-Ketosteroide (Androsteron, Ätiocholanolon und Dehydroepiandrosteron). Die zweite Fraktion wird mit $30 \mathrm{ml}$ Benzol $+0,5 \mathrm{ml}$ Äthanol und dann $30 \mathrm{~m} l$ Benzol $+3 \mathrm{~m} l$ Äthanol sowie $5 \mathrm{~m} l$ Benzol $+5 \mathrm{~m} l$ Äthanol eluiert. Diese Fraktion enthält 11-Ketoandrosteron, 11-Ketoätiocholanolon, 11- $\beta$-Hydroxy-androsteron, 11- $\beta$-Hydroxy-ätiocholanolon sowie Pregnandiol, Pregnantriol und Pregnantriolon. Nachdem jeweils zu beiden Fraktionen ein interner Standard zugefügt wurde, werden sie zur Trockene eingedampft mit Methanol: Äther $=1: 1(\mathrm{v} / \mathrm{v})$ aufgenommen und in ein Spitzkölbchen überführt. Es wird abermals zur Trockene eingedampft.

\section{Stabilisierung der Steroide}

Zur gaschromatographischen Bestimmung müssen die Steroide in thermisch stabile, flüchtige Derivate überführt werden. Hierzu werden die Hydroxylgruppen entweder in die Silyläther oder in die Acetate überführt. Zur Bestimmung der Androgene und Pregnane verwenden wit die Silylderivate.

Zur Silylierung gibt es zwei Möglichkeiten:

a) Verwendung eines Gemisches von Hexamethyldisilazan (HMDS) und Trimethylchlorsilan (TMCS) nach LuUkxarneN und Mitarbeiter (12),

b) Verwendung von Bistrimethylsilylacetamid (BSA) nach HoRNING und Mitarbeiter (13).

$\mathrm{Da}$ wir uns bereits mit den Vorteilen der Verwendung von BSA an anderer Stelle auseinander gesetzt haben (14), soll hiex nur auf den toutinemäßig durchgeführten Silyliervorgang mit HMDS + TMCS eingegangen werden.

Folgende Schritte werden bei der Silylierung mit HMDS + TMCS unternommen: 
Zum trockencn Stcroidgemisch fügt man 0,5 m/ HMDS, 10proz. an TMCS. Das gut verschlossene Spitzkölbehen wird während ciner Std. bei $65^{\circ}$ inkubiert. Anschließend wird das Silyliergemisch mit trockenem Stickstoff abgeblasen, der Rückstand mit $0,2 \mathrm{ml}$ Tetrachlorkohlenstoff aufgenommen und ein aliquoter Anteil dem Gaschromatographen injiziert.

\section{Gascbromatograpbie}

Die eigentliche Trennung und quantitative Bestimmung crfolgt gaschromatographisch. Als Chromatographen verwenden wir cin Fraktometer F 20 der Fa. Perkin Elmer. Gearbeitet wird mit $2 \mathrm{~m}$ Glassäulc. Als Füllmaterial wird 3proz. XE 60 auf Gaschrom P verwendet. Die gaschromatographischen Bedingungen sind: Säulentemperatur $225^{\circ}$, FID und Verdampferblock auf $260^{\circ}$, Trägergas Stickstoff, ctwa $50 \mathrm{~m} / / \mathrm{Min}$. Die quantitative Auswertung crfolgt mittels internem Standard, wir verwenden Androstendion.

\section{Ergebnisse}

\section{Methode nach ZimmermanN}

1. Untersuchung der Streubreite bei Mehrfachbestimmungen

Unser Augenmerk galt vor allem den Faktoren, die einerseits möglichst konstant gehalten werden sollten, deren Änderung aber innerhalb gewisser Grenzen aus apparativen Gründen nicht verhindert werden kann. Dies sind vor allem zwei Punkte:

a) Einfluß der Reaktionstemperatur

b) Einfluß der Reaktionsdauer.

Zur Untersuchung dieser Einflïsse stellten wir Paralleluntersuchungen mit Harnextrakten an und ließen wie bei der routinemäßigen Untersuchung jeweils zwei Kontrollproben mitlaufen. Wir fanden bei Tempeperaturschwankungen von $\pm 1^{\circ}$ bei einer Inkubationstemperatur von $25^{\circ}$ sowie bei Zeitschwankungen von $\pm 10 \mathrm{Min}$. bei einer Inkubationsdauer von $90 \mathrm{Min}$. Differenzen von nicht mehr als $10 \%$ im Endresultat. Die Veränderungen in der Extinktion waren zwar größer, durch den mitgeführten Standard aber werden diese Veränderungen weitgehend ausgeglichen.

\section{Eichkurven}

Wir stellten für die gaschromatographisch ermittelten Einzelmetabolite Eichkurven auf (Abb. 1). Die verschiedenen Steroide zeigen eine verschieden starke Farbreaktion. Die Differenzen in der Farbintensität variieren vom einfachen bis zu mehr als dem doppelten Wert. Bei einer Summenbestimmung kann diese Tatsache bei Überwiegen eines Metaboliten eine beträchtliche Veränderung des Wertes nach oben und unten bedingen.

\section{Gaschromatographie:}

1. Eichkurven von dem Harn zugefügten Steroiden (Abb. 2)

\section{Streubreite bei Mehrfachbestimmungen}

Die Ergebnisse beider Untersuchungen sind aus $\mathrm{Ab}$ bildung $2 \mathrm{bzw}$. Tabelle 2 ersichtlich. Wir haben hier die eingesetzten Mengen Steroid gegen die bei Mehrfachbestimmungen gaschromatographisch gefundene Fläche eingesetzt, wobei die Fläche des internen Standards gleich 100 gesetzt wurde. Außerdem wurde für
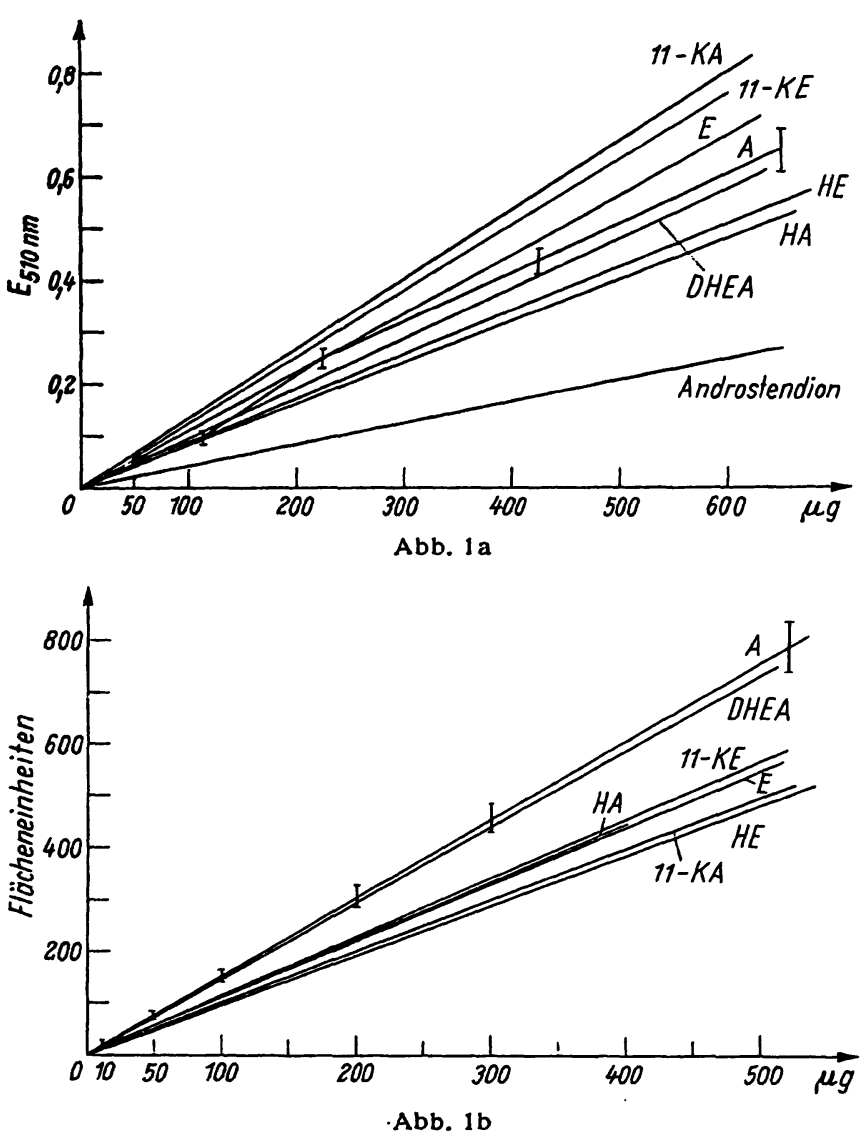

Abb. 1

Steroideichkurven für die Bestimmung nach ZIMMERMANN (a) und mittels Gaschromatographie (b)

Die Eichkurven wurden durch je 5 Parallelbestimmungen mit etwa $10,50,100,200$ und $500 \mu \mathrm{g}$ pro Einzelsteroid erstellt. In den Abbildungen wurde aus Gründen der Übersichtlichkeit nur für Androsteron die Streubreite eingezeichnet. Bei den übrigen Steroiden wurden lediglich die erhaltenen Mittelwerte verbunden aber nicht gesondert einge-

$A=$ Androsteron; $E=$ Ätiocholanolon; DHEA = Dehydroepiandrosteron; $11-\mathrm{KA}=11-\mathrm{Ketoandrosteron;} 11-\mathrm{KE}=11-\mathrm{Ketoäticholano-}$ lon; $\mathrm{HA}=11-\beta$-Hydroxyandrosteron; $\mathrm{HE}=11-\beta-$ Hydroxyätiocholanolon

Tab. 2

Gaschromatographie von Steroiden

Peak-Fläche, erhalten von $100 \mu \mathrm{g}$ Steroid als Silyläther im Vergleich zur Fläche Standard $=100$

a) Steroid direkt silyliert und injiziert, b) Steroid dem Harn zugesetzt extrahiert, säulenchromatographiert und silyliert

\begin{tabular}{|c|c|c|c|}
\hline & $a$ & b & $\begin{array}{c}\text { Ausbeute } \\
\mathrm{b} \text { in } \% \\
\text { von } \mathrm{a}\end{array}$ \\
\hline $\begin{array}{l}\text { Androsteron } \\
\text { Ätiocholanolon } \\
\text { Dehydroepiandrosteron } \\
\text { 11-Keto-androsteron } \\
1 \text { 1-Keto-ätiocholanolon } \\
\text { 1 1- } \beta \text {-Hydroxy-androsteron } \\
1 \text { 1- } \beta \text {-Hydroxy-ätiocholanolon } \\
\text { Pregnandiol } \\
\text { Pregnantriol } \\
\text { Pregnantriolon }\end{array}$ & $\begin{array}{r}155 \\
121 \\
145 \\
122 \\
113 \\
131 \\
109 \\
125 \\
87 \\
81\end{array}$ & $\begin{array}{l}155 \\
110 \\
145 \\
105 \\
110 \\
107 \\
100 \\
115 \\
77 \\
73\end{array}$ & $\begin{array}{r}100 \\
91 \\
100 \\
86 \\
97 \\
82 \\
92 \\
92 \\
88 \\
90\end{array}$ \\
\hline
\end{tabular}

Androsteron Maximum und Minimum eingezeichnet. Die Eichkurve verläuft innerhalb des bei Routineanalysen interessierenden Bereichs geradlinig. Die Streuung beträgt nicht mehr als $\pm 10 \%$. Die bei den einzelnen Steroiden gefundene unterschiedliche Streuung beruht auf verschiedenen Faktoren:

a) Verschieden große, wenn auch in allen Fällen geringe Verluste, bei Inkubation mit $\beta$-Glucuronidase und Solvolyse des Harnes, 
b) unterschiedliche Verluste an der Aluminiumoxidsäule bei der Vorreinigung,

c) unterschiedliche Verluste bei der gaschromatographischen Bestimmung.

Die absolute Größe der Verluste ist aber nicht so wichtig, weit bedeutungsvoller ist die Tatsache, daß alle diese Verluste genau reproduzierbar sind und somit eine auf $\pm 10 \%$ genaue Bestimmung bei Steroidmengen oberhalb von $30 \mu \mathrm{g}$ Steroid/24-Stdn.-Harn zulassen. Bei geringeren Mengen steigt die Streubreite an.

3. Einfluß der Silyliermethode bzw. geringer Abweichungen von der Vorschrift.

Der Einfluß einer Veränderung der Zusammensetzung und Konzentration des Silylierreagenz innerhalb geringer Grenzen auf das Endergebnis ist in Tabelle 3a dargestellt. Veränderungen in der Silylierdauer ersieht man aus Tabelle $3 \mathrm{~b}$, den Einfluß der Temperatur aus

Tab. 3a

Einfluß der Silyliermittelkonzentration auf die erhaltenen Hormonwerte. Relative Peak-Flächen in Prozent des Standards Androstendion

\begin{tabular}{|c|c|c|c|c|c|}
\hline \multirow[b]{2}{*}{ i } & \multicolumn{5}{|c|}{ Silyliermittelmenge pro Ansatz } \\
\hline & $\begin{array}{l}0,5 \\
10\end{array}$ & $\begin{array}{c}1 \\
10\end{array}$ & $\begin{array}{c}0,3 \\
10\end{array}$ & $\begin{array}{l}0,5 \\
20\end{array}$ & $\begin{array}{c}0,5 \\
5\end{array}$ \\
\hline $\begin{array}{l}\text { Androsteron } \\
\text { Attiocholanolon } \\
\text { Dehydroepiandrosteron } \\
\text { 11-Keto-androsteron } \\
\text { 11-Keto-ätiocholanolon } \\
\text { 11- } \beta \text {-Hydroxy-androsteron } \\
\text { 11- } \beta \text {-Hydroxy-ätiocholanolon } \\
\text { Testosteron } \\
\text { Pregnandiol } \\
\text { Pregnantriol } \\
\text { Pregnantriolon } \\
\text { Androstendion }\end{array}$ & $\begin{array}{r}150 \\
121 \\
133 \\
122 \\
107 \\
131 \\
109 \\
118 \\
125 \\
87 \\
81 \\
100\end{array}$ & $\begin{array}{r}157 \\
122 \\
128 \\
117 \\
111 \\
135 \\
104 \\
123 \\
131 \\
84 \\
84 \\
100\end{array}$ & $\begin{array}{r}154 \\
124 \\
129 \\
120 \\
102 \\
133 \\
108 \\
114 \\
120 \\
84 \\
83 \\
100\end{array}$ & $\begin{array}{r}143 \\
116 \\
127 \\
118 \\
103 \\
124 \\
107 \\
115 \\
121 \\
85 \\
78 \\
100\end{array}$ & $\begin{array}{r}148 \\
120 \\
130 \\
127 \\
109 \\
133 \\
104 \\
117 \\
132 \\
92 \\
81 \\
100\end{array}$ \\
\hline
\end{tabular}

Tab. 3 b

Einfluß der Silylierdauer auf die erhaltenen Hormonwerte. Relative Peak-Flächen in Prozent des Standards Androstendion

\begin{tabular}{lcccc}
\hline \multicolumn{1}{c}{ Steroid } & 60 & \multicolumn{4}{c}{ Silylierzeit in Minuten } \\
& & $\mathbf{4 0}$ & $\mathbf{8 0}$ & 100 \\
\hline Androsteron & 150 & 155 & 148 & 149 \\
Ätiocholanolon & 121 & 118 & 120 & 117 \\
Dehydroepiandrosteron & 133 & 130 & 137 & 130 \\
11-Keto-androsteron & 122 & 117 & 125 & \\
11-Keto-ätiocholanolon & 107 & 110 & 109 & \\
11- $\beta$-Hydroxy-androsteron & 131 & 124 & 127 & \\
11- $\beta$-Hydroxy-ätiocholanolon & 109 & 113 & 107 & \\
Testosteron & 118 & 117 & 120 & \\
Pregnandiol & 125 & 125 & 122 & \\
Pregnantriol & 87 & 90 & 84 & 100 \\
Androstendion & 100 & 100 & 100 & 100 \\
Pregnantriolon & 81 & 83 & 80 & \\
\hline
\end{tabular}

Tab. $3 c$

Einfluß der Silyliertemperatur auf die erhaltenen Hormonwerte. Relative Peak-Flächen in Prozent des Standards Androstendion

\begin{tabular}{lccc}
\hline \multicolumn{1}{c}{ Steroid } & $6^{\circ}$ & Temperatur & \\
\hline Androsteron & 150 & 148 & 156 \\
Åtiocholanolon & 121 & 115 & 123 \\
Dehydroepiandrosteron & 133 & 135 & 132 \\
11-Keto-androsteron & 122 & 118 & 120 \\
11-Keto-ätiocholanolon & 107 & 110 & 108 \\
11- $\beta$-Hydroxy-androsteron & 131 & 146 & .128 \\
11- $\beta$-Hydroxy-ätiocholanolon & 109 & 115 & 107 \\
Testosteron & 118 & 115 & 119 \\
Pregnandiol & 125 & 124 & 126 \\
Pregnantriol & 87 & 90 & 83 \\
Pregnantriolon & 81 & 85 & 79 \\
Androstendion & 100 & 100 & 100 \\
\hline
\end{tabular}

Tabelle 3c. In allen Fällen ist der angegebene Wert der Mittelwert aus 3 Parallelbestimmungen, die bei der eingesetzten Menge von $100 \mu \mathrm{g}$ pro Steroid mit einer Genauigkeit von $\pm 7 \%$ übereinstimmen. Die Unterschiede zwischen den Mittelwerten unter verschiedener Reaktionsbedingung liegen innerhalb einer Streubreite von maximal $\pm 15 \%$. Allerdings muß man beachten, $\mathrm{da} \beta$ nicht die absolute Flächengröße angegeben wird, sondern die Fläche Steroịd bezogen auf Fläche Standard $=100$, der von uns verwendete Standard Androstendion wird selbst nicht silyliert, dennoch ist er sicher auch mehr oder weniger empfindlich gegenüber Umweltseinflüssen.

III. Vergleich der nach beiden Methoden erhaltenen Ergebnisse.

Als erstes untersuchten wir bei einer Reihe nach ZIMMERMANN untersuchter Harne die Steroidausscheidung gaschromatographisch und verglichen die so erhaltenen Summen miteinander. Dabei stellten wir dreierlei fest:

1. Bei Erwachsenen stimmen die Werte besser überein als bei Kindern (Tab. 1a).

2. Bei Belastungstests (Tab. 1b) zeigen sich bei der gaschromatographischen Untersuchung weit größere Unterschiede in den Werten als bei Bestimmung der Gesamt-17-Ketosteroide nach der Methode von Z ZmMERMANN.

3. In 3 Fällen konnten wir bei hohen 17-Ketosteroidwerten niedrige Werte für die Einzelmetaboliten feststellen (Tab. 6).

Um die unterschiedlichen Resultate zu erklären, untersuchten wir zuerst den Einfluß der Hydrolyse-Methode (Tab. 4). Die jeweils obere Spalte zeigt die Ergebnisse, die bei enzymatischer Spaltung erhalten würden, gefolgt von Solvolyse und kontinuierlicher Ätherextraktion, die untere Spalte die Ergebnisse nach $\mathrm{HCl}$ Hydrolyse.

Die gaschromatographisch erhaltenen Ergebnisse liegen bei der enzymatischen Methode eindeutig höher als bei der HCl-Spaltung, anders aber die Werte bei der Zimmermann-Reaktion.

Außerdem wurden nach der Säulenchromatographie aus einem Teil des Eluates die Steroide gaschromatographisch und aus einem $z$ weiten Teil nach der Methode von ZIMMERMANN ermittelt. Die säulenchromatographische Vorreinigung ergab aber keine wesentliche Veränderung der Werte im Vergleich zu denen, die ohne Vorreinigung erstellt worden waren.

Daraufhin untersuchten wir bei zwei Harnen jeden Einzelschritt mit der Methode nach ZimmermanN und mittels Gaschromatographie (Tab. 5).

Aus dieser Tabelle ersehen wir, daß die Summe der gaschromatographisch ermittelten 17-Ketosteroide recht gut mit der kolorimetrisch ermittelten Summe übereinstimmt, wenn wir in beiden Fällen die Konjugate enzymatisch und daran anschließend durch Solvolyse spalten. 
$\mathrm{Da}$ aber diese Werte wesentlich niedriger sind als die nach $\mathrm{HCl}$-Hydrolyse und Zimmermann-Reaktion erhaltenen Werte oder die nach ZimmermanN-Pontius oder BIRKET-SMITH erhaltenen Resultate, wollten wir feststellen, wo die Verluste auftreten und ob es sich lediglich um einen Verlust der unspezifischen Chromogene oder auch um einen Verlust an Steroiden handelt. Ein Aliquot des Harnes wurde mit $\beta$-Glucuronidase inkubiert, dann der Hydrolyse mit $\mathrm{HCl}$ unterworfen und der Ätherextrakt der Zimmermann-Reaktion unterworfen. Es fand sich nahezu der gleiche Wert wie vorher.
Dann wurden aus einem Aliquot die Steroide nach Glucuronidasespaltung mit Äther extrahiert und aus dem verbliebenen Harn nach der Zimmermann-Methode die 17-Ketosteroide bestimmt, ebenso aus dem Ätherextrakt. Die Summe der beiden Werte entsprach wiederum dem ursprünglichen Wert. Nach der Solvolyse bei kontinuierlicher Ätherextraktion wurde wiederum der Ätherextrakt und der nun unserer Meinung nach von den Steroiden befreite Harn einer Zimmermann-Reaktion unterworfen. Hierbei fanden wir eine weitaus stärkere Reaktion in der verbliebenen wäßrigen Phase als erwartet. Die Summe der Werte

Tab. 4

Vergleichende Untersuchung des Hydrolyseverfahrens auf die 17-Ketosteroid-Bestimmung mit dem Gaschromatographen und nach ZIMMERMANN. Hormonausscheidung in $\mu \mathrm{g} / \mathrm{Tag}$

\begin{tabular}{|c|c|c|c|c|c|c|c|c|c|c|c|c|c|c|}
\hline \multirow{2}{*}{ Name } & \multirow{2}{*}{$\begin{array}{c}\text { Ge- } \\
\text { schlecht }\end{array}$} & \multirow{2}{*}{$\underset{\text { mit }}{\text { Hydrolyse }}$} & \multirow{2}{*}{ 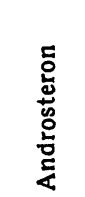 } & \multirow{2}{*}{ 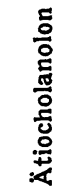 } & \multirow{2}{*}{ 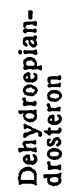 } & \multirow{2}{*}{ 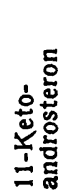 } & \multirow{2}{*}{ 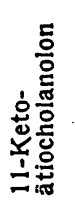 } & \multirow{2}{*}{ 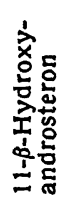 } & \multirow{2}{*}{ 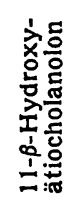 } & \multirow{2}{*}{ 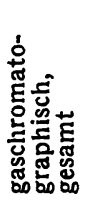 } & \multicolumn{3}{|c|}{$\begin{array}{l}\text { 17-Ketosteroide nach } \\
\text { ZIMMERMANN }\end{array}$} & \multirow{2}{*}{ 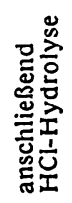 } \\
\hline & & & & & & & & & & & & $\begin{array}{r}\text { tion } \\
2\end{array}$ & Gesamt & \\
\hline K. A. & $\sigma^{\circ}$ & $\beta$-Glucuronidase & $\begin{array}{l}2330 \\
2200\end{array}$ & $\begin{array}{l}936 \\
880\end{array}$ & $\begin{array}{r}109 \\
11\end{array}$ & $\begin{array}{r}260^{\circ} \\
46\end{array}$ & $\begin{array}{r}340 \\
67\end{array}$ & $\begin{array}{r}140 \\
21\end{array}$ & $\begin{array}{l}98 \\
14\end{array}$ & $\begin{array}{l}4213 \\
3239\end{array}$ & $\begin{array}{l}3100 \\
5070\end{array}$ & $\begin{array}{r}620 \\
1220\end{array}$ & $\begin{array}{l}3802 \\
6280\end{array}$ & 2535 \\
\hline Sch. H. & 우 & $\underset{\mathrm{HCl}}{\beta \text {-Glucuronidase }}$ & $\begin{array}{l}730 \\
670\end{array}$ & $\begin{array}{l}1210 \\
1040\end{array}$ & $\begin{array}{l}480 \\
260\end{array}$ & $\begin{array}{r}370 \\
58\end{array}$ & $\begin{array}{r}280 \\
34\end{array}$ & $\begin{array}{r}111 \\
17\end{array}$ & $\begin{array}{l}76 \\
12\end{array}$ & $\begin{array}{l}3257 \\
2091\end{array}$ & $\begin{array}{l}2840 \\
4325\end{array}$ & $\begin{array}{l}1100 \\
1235\end{array}$ & $\begin{array}{l}4013 \\
6773\end{array}$ & 4384 \\
\hline T. B. & ㅇ & $\underset{\text { HCl }}{\beta \text {-Glucuronidase }}$ & $\begin{array}{l}1550 \\
1500\end{array}$ & $\begin{array}{l}1700 \\
1600\end{array}$ & $\begin{array}{r}730 \\
54\end{array}$ & $\begin{array}{r}450 \\
34\end{array}$ & $\begin{array}{r}420 \\
66\end{array}$ & $\begin{array}{r}320 \\
32\end{array}$ & $\begin{array}{r}240 \\
12\end{array}$ & $\begin{array}{l}5410 \\
3298\end{array}$ & $\begin{array}{l}3840 \\
3718\end{array}$ & $\begin{array}{r}965 \\
1014\end{array}$ & $\begin{array}{l}5095 \\
5540\end{array}$ & 1521 \\
\hline
\end{tabular}

Tab. 5

Untersuchung der Einzelschritte im Analysengang mit der Methode nach ZIMMERMANN und der Gaschromatographie an zwei Harnen. Angaben in $\mu \mathrm{g} /$ Tag. $\mathrm{I}=$ Proband $\mathrm{K}$. W. $\sigma^{\prime}, \mathrm{II}=$ Proband $\mathrm{N}$. U. $\sigma^{\prime \prime}$

\begin{tabular}{|c|c|c|c|c|}
\hline & $\begin{array}{c}\beta \text {-Glucuroni- } \\
\text { dase }\end{array}$ & Sulfatase & Bestimmung nach ŻIMMERMANN & \\
\hline $\begin{array}{l}\text { I. } \\
\text { Androsteron } \\
\text { Ätiocholanolon } \\
\text { Dehydroepiandrosteron } \\
\text { 11-Keto-androsteron } \\
11-\text { Keto-ätiocholanolon } \\
\text { 11- } \beta \text {-Hydroxy-androsteron } \\
11-\beta \text {-Hydroxy-ätiocholanolon }\end{array}$ & $\begin{array}{l}372 \\
596 \\
57 \\
231 \\
775 \\
165 \\
237 \\
\end{array}$ & $\begin{array}{l}485 \\
225 \\
= \\
= \\
\end{array}$ & \multirow[t]{2}{*}{$\begin{array}{l}\text { Harn direkt nach ZIMMERMANN } \\
\text { Harn nach a) ZiMMERMANN-PONTIUS } \\
\text { b) BIRKET-SMITH } \\
\text { Harn nach } \beta \text {-Glucuronidaseinkubation } \\
\text { Harn nach Atherextraktion } \\
\text { Harn nach Solvolyse und kontinuierlicher Ätherextraktion } \\
\text { Harn nach Ätherextraktion und Âtherextrakt } \\
\text { Harn nach Solvolyse und Summe der Ätherextrakte } \\
\text { Summe des Waschwassers }\end{array}$} & \multirow[t]{3}{*}{$\begin{array}{r}6597 \\
6515 \\
6470 \\
6437 \\
4114 \\
2436 \\
6406 \\
5858 \\
200\end{array}$} \\
\hline ZIMMERMANN & $\begin{array}{l}2433 \\
2292 \\
\end{array}$ & $\begin{array}{r}710 \\
1130 \\
\end{array}$ & & \\
\hline $\begin{array}{l}\text { Gaschromatographie } \\
\text { ZIMMERMANN }\end{array}$ & $\begin{array}{l}3143 \\
3422\end{array}$ & & \multirow{4}{*}{$\begin{array}{l}\text { Harn direkt } \\
\text { Härn nach a) ZIMMERMANN-PoNTIus } \\
\text { b) BiRKET-SMITH } \\
\text { Harn nach Inkubation } \\
\text { Harn nach Ätherextraktion } \\
\text { Harn nach Solvolyse und kontinuierlicher Ätherextraktion } \\
\text { Summe des Waschwassers } \\
\text { Harn nach Âtherextraktion und Ätherextrakt } \\
\text { Harn nach Solvolyse und Summe der Ätherextrakte }\end{array}$} & \\
\hline $\begin{array}{l}\text { Androsteron } \\
\text { AI. } \\
\text { Átiocholanolon } \\
\text { Dehydroepiandrosteron } \\
\text { 11-Keto-androsteron } \\
\text { i1-Keto-ätiocholanolon } \\
11-\beta \text {-Hydroxy-androsteron } \\
\text { 11- } \beta \text {-Hydroxy-ätiocholanolon }\end{array}$ & $\begin{array}{r}328 \\
1151 \\
42 \\
244 \\
495 \\
144 \\
351 \\
\end{array}$ & $\begin{array}{l}231 \\
174 \\
246 \\
= \\
=\end{array}$ & & \multirow[t]{3}{*}{$\begin{array}{r}8710 \\
8600 \\
8540 \\
8490 \\
4820 \\
3505 \\
200 \\
8769 \\
8152\end{array}$} \\
\hline ZIMMERMANN & $\begin{array}{r}2755 \\
3949 \\
\end{array}$ & $\begin{array}{l}651 \\
698 \\
\end{array}$ & & \\
\hline $\begin{array}{l}\text { Gaschromatographie } \\
\text { ZIMMERMANN }\end{array}$ & $\begin{array}{l}3406 \\
4647\end{array}$ & & & \\
\hline
\end{tabular}

Tab. 6

Niedrige Ausscheidung der Einzelmetabolite bei hohem Wert der 17-Ketosteroide nach ZIMMERMANN in $\mu \mathrm{g} / \mathrm{Tag}$

\begin{tabular}{|c|c|c|c|c|c|c|c|c|c|c|c|c|}
\hline Name & Alter & $\begin{array}{c}\text { Ge- } \\
\text { schlecht }\end{array}$ & 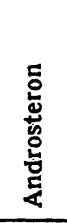 & 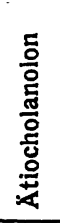 & 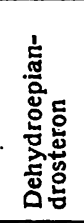 & 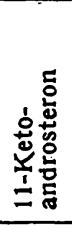 & 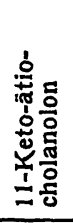 & 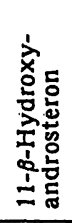 & 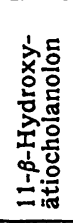 & 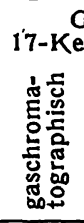 & 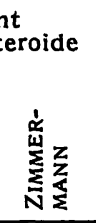 & 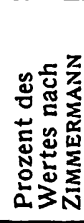 \\
\hline $\begin{array}{l}\text { F. A. } \\
\text { H. K. }\end{array}$ & $\begin{array}{l}37 \text { Jahre } \\
\text { I } 28 \text { Jahre } \\
\text { II }\end{array}$ & 옹 & $\begin{array}{l}160 \\
183 \\
147\end{array}$ & $\begin{array}{l}43 \\
93 \\
73\end{array}$ & $\begin{array}{r}16 \\
5 \\
5\end{array}$ & $\begin{array}{l}12 \\
27 \\
78\end{array}$ & $\begin{array}{l}23 \\
21 \\
13\end{array}$ & $\begin{array}{r}38 \\
7 \\
9\end{array}$ & $\begin{array}{l}16 \\
21 \\
19\end{array}$ & $\begin{array}{l}308 \\
357 \\
344\end{array}$ & $\begin{array}{l}13560 \\
14900 \\
15100\end{array}$ & $\begin{array}{l}2,3 \\
2,4 \\
2,3\end{array}$ \\
\hline
\end{tabular}


beider Ätherextrakte und der wäßrigen Phase nach erfolgter Extraktion ergab wiederum einen Wert, der vom ursprünglichen Wert nicht sehr verschieden ist.

Nun war noch zu klären, ob es sich bei der Farbreaktion der wäßrigen Pbase um Steroide oder lediglich um unspezifische Chromogene handelt. Wir unterzogen den bereits extrahierten Harn wie vorher einer salzsauren Hydrolyse und daran anschließend einer Ätherextráktion. Der Ätherextrakt wurde zweigeteilt und mit einem Teil eine Farbreaktion mit Dinitrobenzol mit dem anderen Teil eine gaschromatographische Analyse durchgeführt. Die Farbreaktion bestätigte den ersten Befund, während im Gaschromatogramm keine Zacken auffindbar waren. Somit ist bewiesen, daß es sich bei diesen Chromogenen nicht um Steroide handelt. Dieser Befund stimmt auch recht gut mit dem Befund von CuRtius (10) überein, daß Dehydroepiandrosteronsulfat nach der angegebenen Solvolyse vollkommen gespalten wird und im Ätherextrakt vorliegt.

\section{Diskussion}

Wir untersuchten, warum die Summe der gaschromatographisch ermittelten 17-Ketosteroide nicht mit dem nach der Methode von ZimmermanN erhaltenen Wert übereinstimmt und wie genau die gaschromatographische Methode ist.

Wir konnten zeigen, daß die nach beiden Methoden erhaltenen Summen bei enzymatischer Spaltung der Glucuronide und anschließender schonender Solvolyse und kontinuierlicher Ätherextraktion übereinstimmen. Außerdem konnten wir feststellen, daß bei der heißen Säurehydrolyse die gaschromatographisch nachweisbaren 17-Ketosteroide abnehmen, der Wert nach ZiMmermanN höher ist als nach schonender Solvolyse bei Raumtemperatur.
Ein Harn wurde nun nach der schonenden Methode von Steroiden befreit und dann der heịen Säurehydrolyse unterworfen. In dem Ätherextrakt konnten gaschromatographisch keine Steroide nachgewiesen werden, wohl aber eine starke Zimmermann-Reaktion. Damit scheint nun bewiesen, daß bei der Säurehydrolyse Stoffe entstehen, die ätherextrahierbar sind und eine Zimmermann-Reaktion geben. Außerdem konnten wir mit unserer Untersuchung zeigen, $\mathrm{da} \beta$ bei der Ätherextraktion und beim Waschen des Ätherextraktes so gut wie keine Verluste auftreten, da wir keine Farbreaktion erhalten, wenn wir die Waschwässer einer Zimmermann-Reaktion unterwerfen. Die Verluste, die aber bei der Säulenchromatographie sowie bei der gaschromatographischen Bestimmung entstehen, sind stets reproduzierbar und somit durch eine Eichkurve eliminierbar.

Die Unterschiede der Summe der gaschromatographisch bestimmten 17-Ketosteroide und der nach der Zimmermann-Methode ermitteltem Wert konnten wie folgt geklärt werden:

1. Substanzen, die bei der heißen Säurehydrolyse entstehen, sind ätherextrahierbar und geben mit Dinitrobenzol eine Farbreaktion.

2. Die verschiedenen Steroide geben eine unterschiedliche Farbreaktion, dieser Faktor ist bei einer Summenbestimmung aber nicht eliminierbar. Für die gaschromatographische Bestimmung der 17-Ketosteroide konnte gezeigt werden, daß 1 . die angegebenen Hydrolyseverfahren wirklich schonend sind und sämtliche Konjugate erfaßt werden, 2. sämtliche bei der Präparation auftretenden Fëhler durch eine für jedes Steroid aufgestellte Eichkurve eliminierbar sind und somit die Genauigkeit einer gaschromatographischen Bestimmung für Mengen von mehr als $30 \mu \mathrm{g} / 24-\mathrm{Stdn}$.Harn bei $\pm 10 \%$ liegt.

\section{Literatur}

1. Dingemanse, E., L. G. Huis in't Veld und S. L. HarthoghKaTz, J. Clin. Endocr., Springfield, 12, 66 (1952). - 2. CREMER, E. und H. NAU, Naturwissenschaften, 55, 651 (1968). - 3. ZIMMERMANN, W., Chemische Bestimmungsmethoden von Steroidhormonen in Körperflüssigkeiten, Springer-Verlag Berlin-Göttingen-Heidelberg (1955). - 4. Lroyd, C. W., J. Loвotsky, E. J. Segre, T. Kobayaski, M. L. Taymor und R. E. Batt, J. Clin. Endocr., Springfield, 26, 314 (1966). - 5. ZimmermanN, W. und D. Ponrius, Hoppe-Seyler's Z. physiol. Chem., 297, 157 (1954). 6. BrRket-Smith, E., Acta endocr., Kh'vn, 14, 33 (1953). 7. Neinhoeffler, O., $K$. Thewalt und $W$. Zimmermann,
Hoppe-Seyler's Z. physiol. Chem., 323, 116 (1961). - 8. Gibson, J. G. und K. A. Evelyn, J. Clin. Invest., 17, 153 (1938). 9. AlleN, W. M., J. Clin. Endocr., Springfield, 10, 91 (1950). 10. Brown, J. B., Biochem. J., 60, 185 (1955). - 11. Curtius, H. CH. und M. MülleR, J. Chromatogr., 30, 410 (1967). 12. Luukkainen, T., W. J. A. van den Heuvel, E. O. A. HaAhti und E. C. Horning, Biochim. biophysica Acta, Amsterdam, 52, 599 (1961). - 13. Horning, E. C., M. G. Horning, N. IkEKAwA, E. M. Chambaz, P. I. JaAkonmakx und C. H. W. Brooks, J. Gaschromatogx., 5, 283 (1967). - 14. GleispACH, H., Z. analyt. Chem., 243, 294 (1968).
Dr. H. Gleispach A-6020 Innsbruck Anichstr. 35 